\title{
Estesioneuroblastoma: relatos de casos e revisão de literatura
}

\section{Esthesioneuroblastoma: Case Reports and Literature Review}

\author{
Marcelo Lemos Vieira da Cunha ${ }^{1}$ Ronaldo Rodrigues de Oliveira ${ }^{2}$ Débora de Césaro ${ }^{2}$ \\ Alexandre Schneider ${ }^{2}$ Lauro Melo ${ }^{2}$ \\ ${ }^{1}$ Neurocirurgião do Hospital Regional do Oeste, Chapecó, SC, Brasil; \\ Membro Titular da Sociedade Brasileira de Neurocirurgia, São Paulo, \\ Brasil \\ Address for correspondence Marcelo Lemos Vieira da Cunha, MD, Rua \\ Rui Barbosa, 93E-ap.501, Centro, Chapecó, SC, Brasil, CEP 89801-040 \\ (e-mail: marcelolvc@yahoo.com.br).
}

${ }^{2}$ Acadêmicos de Medicina da Universidade Comunitária Regional de Chapecó (Unochapecó), Chapecó, Santa Catarina, Brasil

Arq Bras Neurocir 2015;34:153-156

\section{Resumo \\ Palavras Chave \\ - estesioneuro- blastoma \\ - base do crânio \\ - prognóstico}

O estesioneuroblastoma (ENB) é uma neoplasia rara que atinge o neuroepitélio olfativo, comumente invadindo os seios paranasais, a base do crânio e a região orbitária. Apresenta metástase em 10 a 30 \% dos casos. Distribui-se de forma bimodal para a idade, principalmente dos 11 aos 20 anos e dos 51 aos 60 anos, sem influência do sexo e raça do indivíduo. A ínfima quantidade de casos relatados resulta em dúvidas quanto ao melhor tratamento a ser dispensado ao paciente. Relatamos três casos, todos Kadish $\mathrm{C}$, tornando o tratamento e o prognóstico um desafio à prática neurocirúrgica.

The esthesioneuroblastoma (ENB) is a rare neoplasm that affects the olfactory neuroepithelium, commonly invading the paranasal sinuses, skull base and the orbital region. Shows metastasis in $10 \%$ to $30 \%$ of cases. Distributed in a bimodal shape for the age, from 11 to 20 years and 51 to 60 years especially, with no influence of gender and race of the individual. The tiny amount of reported cases result in doubt about the best treatment to be dispensed to the patient. We report three cases, all Kadish C, making treatment and prognosis a challenge to neurosurgery.

\section{Introdução}

O estesioneuroblastoma (ENB), também conhecido como neuroblastoma olfatório, é uma neoplasia rara que acomete o neuroepitélio olfativo, com frequente invasão da região dos seios paranasais, base do crânio e região orbitária. ${ }^{1-4} \mathrm{O}$ ENB representa $6 \%$ dos casos de tumor na cavidade nasal e seios paranasais e menos de $1 \%$ do total de tumores malignos. ${ }^{1}$ Há relatos de metástase em 10 a 30\% dos casos, sendo mais comum para os linfonodos cervicais. No entanto, metástases mais distantes podem ocorrer afetando cérebro, traqueia, pulmões, coração, fígado e ossos. ${ }^{4}$

A raridade deste tipo de neoplasia fica evidente após revisões na literatura sobre o tema, visto que foram referidos

\section{Recebido}

August 25, 2014

Aprovado

March 31, 2015
DOI http://dx.doi.org/

10.1055/s-0035-1554048. ISSN 0103-5355.
Copyright $\odot 2015$ by Thieme Publicações License terms Ltda, Rio de Janeiro, Brazil 
menos de mil casos da doença desde a sua primeira descrição por Berger et al. em 1924 no artigo “L'esthesioneuropitheliome olfactif". 1

Constata-se que o surgimento da doença se distribui de forma bimodal para a idade, sendo mais comum entre os $11 \mathrm{e}$ 20 anos e entre os 51 e 60 anos, ${ }^{2,3}$ apesar de já terem sido relatados casos em idades mais extremas, como bebês antes dos 18 meses de idade e idosos na nona década de vida. ${ }^{1}$ Também é notório que o sexo e a raça do indivíduo não influenciam no acometimento da referida neoplasia. ${ }^{1}$

\section{Relato dos Casos}

Caso 1: paciente masculino, 40 anos, com procura direta de nosso serviço por cefaleia refratária ao tratamento clinico. À anamnese dirigida, relatou congestão nasal há 6 meses e três episódios de epistaxe nos últimos 3 meses. Ao exame, apresentou-se sem déficit de consciência, sem déficits focais neurológicos e sem linfonodomegalia cervical. À tomografia computadorizada (TC) do tórax, mostrou-se dentro da normalidade. À TC de crânio, foi evidenciada lesão expansiva de base craniana anterior com invasão dos seios paranasais. À complementação com ressonância magnética (RM) de encéfalo, a lesão foi classificada em Kadish C ( - Fig. 1). Submetido à biópsia transnasal, o paciente recebeu diagnóstico de estesioneuroblastoma (Hyams grau III) e foi indicado para ressecção neurocirúrgica. No entanto, paciente não concordou com tratamento neurocirúrgico e realizou apenas tratamento radioterápico. Após 2 meses de seguimento, manteve episódios de epistaxe, sem outras queixas.

Caso 2: paciente masculino, 53 anos, encaminhado ao nosso serviço após verificação de exoftalmia à esquerda com TC de crânio evidenciando lesão expansiva de fossa anterior craniana há 2 meses. Ao exame, apresentou-se sem déficit de consciência, com exoftalmia com restrição de motilidade

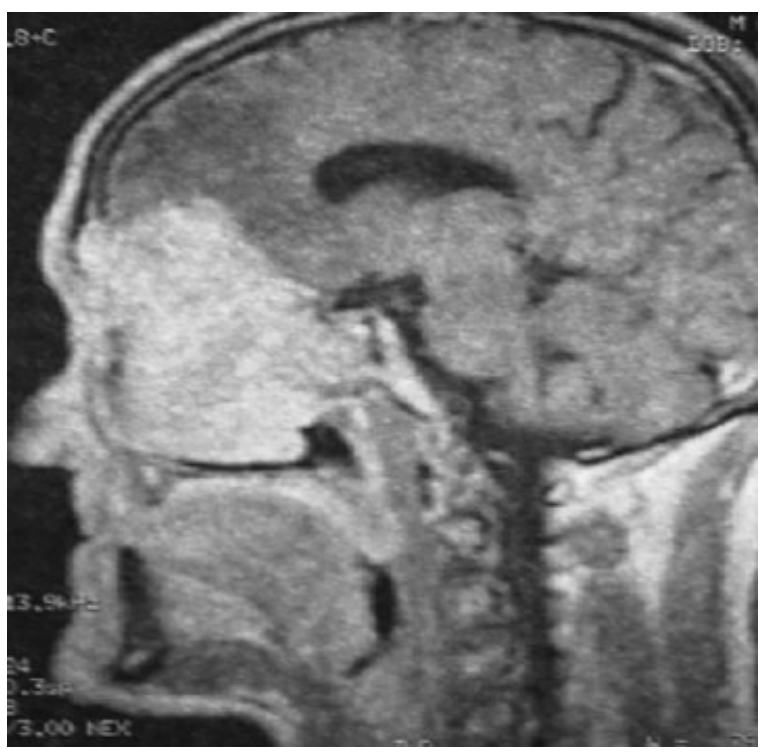

Fig. 1 Lesão hiperdensa em corte sagital na sequência T1 de ressonância magnética de encéfalo após infusão de contraste associado ao edema vasogênico frontal. ocular à esquerda, e borramento visual corroborado com campimetria computadorizada. À complementação com RM, recebeu classificação de Kadish C. À TC do tórax, mostrou-se sem anormalidades. $O$ paciente inicialmente apresentou restrição ao tratamento neurocirúrgico. Após 3 meses, com piora clínica - cegueira à esquerda, borramento visual à direita, piora da exoftalmia à esquerda - e três episódios de crise convulsiva, concordou com tratamento cirúrgico. À TC de crânio, foi demonstrado aumento lesional (-Fig. 2). A RM estava indisponível no pré-operatório. Submetido à ressecção de lesão e reconstrução de base craniana, o paciente não apresentou novos déficits focais (-Fig. 3). O estudo anatomopatológico evidenciou Hyams grau IV. Submetido à radioterapia, o paciente evoluiu para óbito 6 meses após tratamento cirúrgico e radioterápico.

Caso 3: paciente feminino, 50 anos, com 4 meses de tratamento por sinusite crônica e 1 mês de episódios frequentes de epistaxes. À TC de crânio, evidenciou-se lesão expansiva de cavidade nasal. À complementação com RM (-Fig. 4), recebeu classificação de Kadish C. A ressecção completa via transcraniana foi realizada sem déficits pósoperatórios (-Figs. 5 e 6 ). 0 estudo anatomopatológico mostrou-se compatível com estesioneuroblastoma, Hyams grau III. A paciente foi encaminhada para tratamento radioterápico complementar.

\section{Discussão}

O estesioneuroblastoma apresenta sintomas inespecíficos e muitas vezes comuns a outras patologias da cavidade nasal, ${ }^{5}$

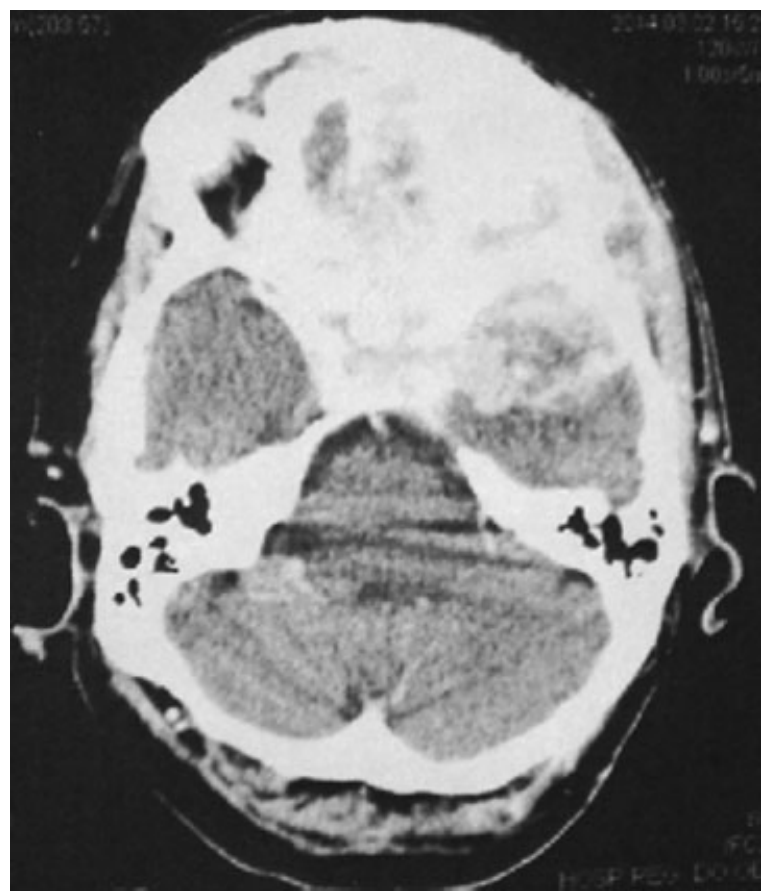

Fig. 2 Corte axial de tomografia computadorizada de crânio após infusão de contraste endovenoso evidenciando lesão expansiva que ocupa toda a base anterior do crânio, órbita esquerda e fossa média esquerda. 


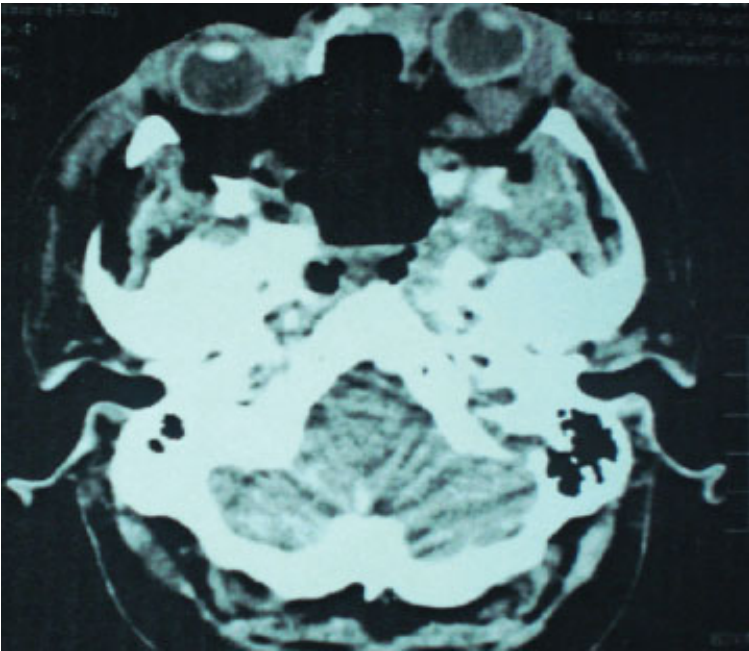

Fig. 3 Tomografia computadorizada de crânio evidenciando ampla ressecção lesional em corte axial.

como a rinossinusite crônica. ${ }^{6}$ Pacientes relatam a progressão dos sintomas no período de meses ou anos. ${ }^{2}$ Juntamente com os sintomas inespecíficos, o período de tempo dificulta o diagnóstico, que é realizado geralmente em estágios mais avançados. ${ }^{7,8}$ Os sintomas mais frequentes são epistaxe, obstrução nasal unilateral e rinorreia. Distúrbios visuais, cefaleia e hiposmia também são relatados. ${ }^{5}$

Os exames radiológicos mais comumente utilizados são a TC, que permite avaliar o envolvimento ósseo, e, complementarmente, a RM, que permite determinar a extensão exata do tumor. ${ }^{7}$ Os três pacientes citados foram submetidos inicialmente à TC de crânio, que evidenciou lesão expansiva em todos os casos. Posteriormente, foi realizada RM de encéfalo no pré-operatório. Segundo a literatura, como o ENB se origina na porção superior da cavidade nasal, rapidamente pode invadir os seios paranasais e a fossa craniana anterior, que possibilita a disseminação via meníngea para o encéfalo e medula. ${ }^{7}$ Este tipo de disseminação foi verificada nos casos 2 e 3.

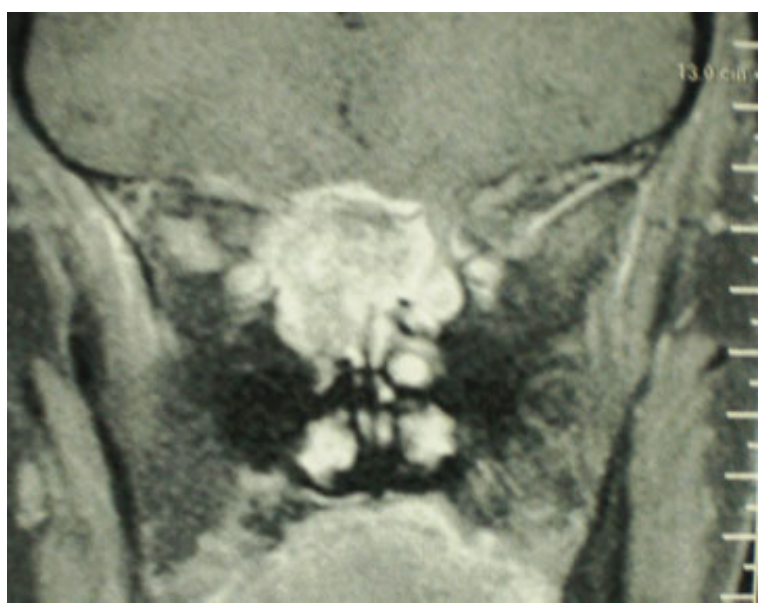

Fig. 4 Corte coronal de ressonância magnética de encéfalo com lesão hipercaptante em T1 com invasão de órbita direita e intracraniana.

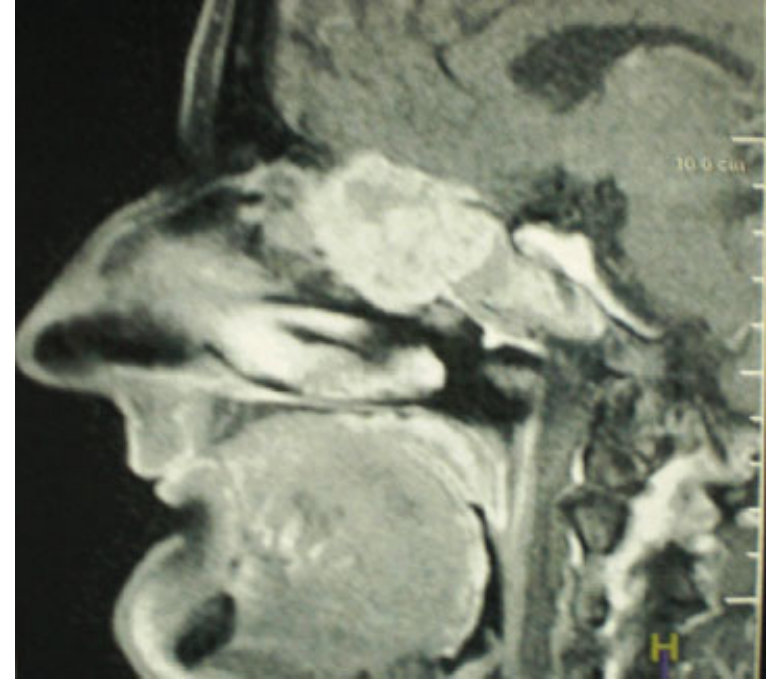

Fig. 5 Corte sagital de ressonância magnética de encéfalo em T1 com captação após contraste.

Outro método utilizado para se chegar a um diagnóstico mais preciso é o uso de exames imuno-histoquímicos e histológicos, uma vez que eles ajudam a diferenciar este tumor do melanoma, linfoma, tumor de glândulas salivares menores ou ainda do carcinoma nasossinusal indiferenciado. As células do estesioneuroblastoma possuem características basicamente homogêneas, sendo arredondadas, apresentando rosetas de Homer-Wright ou rosetas de Flexner-Wintersteiner. Suas características são determinantes tanto para neoplasias neuronais como neuroendócrinas. ${ }^{9}$ Nos casos relatados, foi confirmado o diagnóstico de ENB pelos achados histológicos; o primeiro, através da biópsia da lesão, e os demais, pela macro e microscopia da peça ressecada.

A classificação do ENB pode ser realizada ${ }^{10}$ através da escala de Kadish, ${ }^{11}$ que se baseia na extensão do tumor

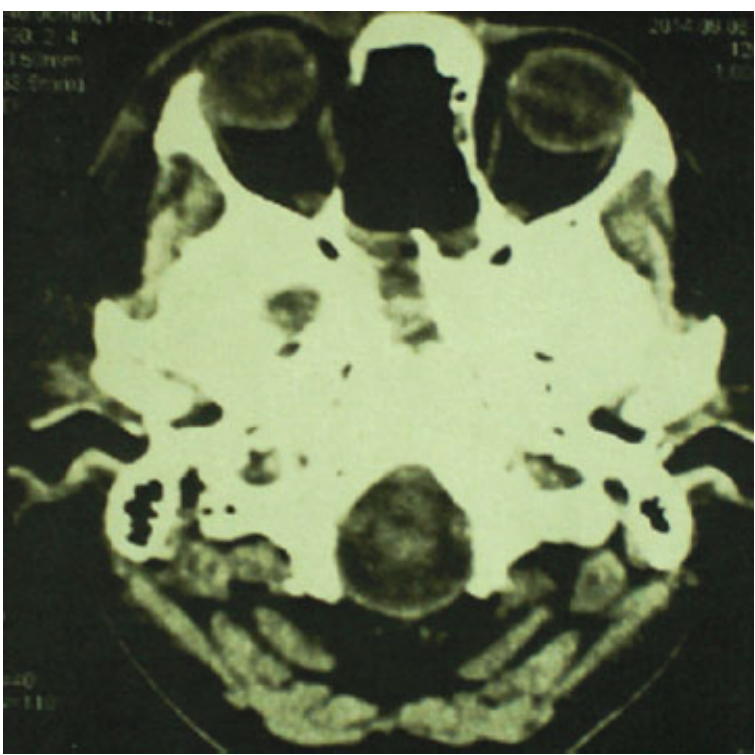

Fig. 6 Tomografia computadorizada de crânio após ressecção completa de lesão da base craniana anterior. 
primário. ${ }^{12} \mathrm{O}$ grupo A é formado pelos pacientes que apresentam o tumor restrito na cavidade nasal; o grupo B, por aqueles em que o tumor ocupa a cavidade nasal e os seios paranasais; e o grupo $\mathrm{C}$ - caso dos três pacientes aqui relatados -, pelos que apresentam tumores que vão além dos seios paranasais ${ }^{7,11,13}$ (base de crânio, órbita ou metástases). ${ }^{13}$

A classificação histopatológica de Hyams é utilizada para diagnóstico definitivo, pois possibilita a diferenciação com outros tipos de tumores ${ }^{12}$ como carcinomas neuroendócrinos e carcinomas sinonasais indiferenciados. ${ }^{7}$ Esta classificação varia do grau I ao IV, dependendo da diferenciação celular. ${ }^{12}$ No grau I, os tumores são caracterizados por células tumorais com núcleos uniformes, uma matriz fibrilar proeminente e ausência de atividade mitótica, pleomorfismo nuclear ou necrose, ${ }^{10}$ indicando bom prognóstico para o paciente. ${ }^{8}$ No grau IV, os tumores se apresentam com aumento da atividade mitótica, marcado pleomorfismo nuclear, ausência de matriz fibrilar e rosetas, e frequente necrose. ${ }^{10}$ Todos os pacientes que apresentam grau IV falecem pela doença. ${ }^{8}$

O tratamento para o estesioneuroblastoma (ENB) tem evoluído de excisão cirúrgica extracraniana para regimes de tratamento multimodalidade. ${ }^{4}$ Entretanto, não há consenso sobre o melhor tratamento dos estesioneuroblastomas. ${ }^{14}$ A ressecção em bloco mostra-se como o melhor método para um procedimento seguro, ${ }^{4}$ sendo portanto indicada para os casos de grau $\mathrm{C}$ de Kadish considerados operáveis. ${ }^{15}$ A escolha dessa tradicional técnica justifica-se por sua maior segurança em margens cirúrgicas livres e, por consequência, menor índice de recidivas. ${ }^{8}$ Além disso, a abordagem convencional permite uma reconstrução mais confiável da base do crânio. ${ }^{8}$

Segundo Lund et al., a ressecção craniofacial associada à radioterapia é o tratamento padrão-ouro, ${ }^{5}$ visto que o ENB tem sido descrito como um tumor radiossensível. ${ }^{16}$ Como os três pacientes dos casos aqui relatados foram enquadrados no grau C de Kadish, foi indicada para eles a ressecção cirúrgica associada à radioterapia, corroborando as indicações da literatura. Entretanto, o paciente do primeiro caso não aceitou o tratamento proposto.

Segundo Pedruzzi et al., ${ }^{17}$ o estesioneuroblastoma é um tumor altamente agressivo com alta taxa de recidiva local, de até $50 \%$. O grau histológico, o estado clínico e a presença de metástases são fatores prognósticos. ${ }^{1}$ A sobrevida após a recidiva é de aproximadamente 12 meses, e a maioria das recorrências acontece nos primeiros anos após o tratamento. ${ }^{18}$

O estesioneuroblastoma mostra-se como uma neoplasia rara. Devido à presença de sintomas inespecíficos e muitas vezes comuns a outras patologias da cavidade nasal e adjacências, o diagnóstico é tardio. É necessária a inclusão do ENB como diagnóstico diferencial em casos com sinais e/ou sintomas nasais de tratamento refratário. Assim, com um maior índice de suspeição, há mais subsídios para que se tente modificar a agressividade da doença e o comprometimento global do paciente.

\section{Referências}

1 Castelnuovo PG, Delù G, Sberze F, et al. Esthesioneuroblastoma: endonasal endoscopic treatment. Skull Base 2006;16(1):25-30

2 Gondim J, Ramos F Jr, Azevedo J, Carrero FP Jr, Tella OI Jr. Esthesioneuroblastoma: case report. Arq Neuropsiquiatr 2002; 60(2-A):303-307

3 Oskouian RJ Jr, Jane JA Sr, Dumont AS, Sheehan JM, Laurent JJ, Levine PA. Esthesioneuroblastoma: clinical presentation, radiological, and pathological features, treatment, review of the literature, and the University of Virginia experience. Neurosurg Focus 2002;12(5):e4

4 Resto VA, Eisele DW, Forastiere A, Zahurak M, Lee DJ, Westra WH. Esthesioneuroblastoma: the Johns Hopkins experience. Head Neck 2000;22(6):550-558

5 Lund VJ, Howard D, Wei W, Spittle M. Olfactory neuroblastoma: past, present, and future? Laryngoscope 2003;113(3):502-507

6 Mendonça VF, Carvalho ACP, Freitas E, Boasquevisque EM. Tumores malignos da cavidade nasal: avaliação por tomografia computadorizada. Radiol Bras 2005;38(3):175-180

7 Caeiro C, Jaraquemada T, Augusto I, Sarmento C, Damasceno M. Estesioneuroblastoma: Caso Clínico e Revisão da Literatura. Arq Med 2008;22(1):21-23

8 Monteiro EMR, Lopes MG, Santos ER, et al. Endoscopic treatment of esthesioneuroblastoma. Braz J Otorhinolaryngol 2011;77(2): 171-177

9 Ferreira MCF, Tonoli C, Varoni ACC, et al. Estesioneuroblastoma. Rev Cienc Med 2007;3(16):193-198

10 Barnes L, Kapadia SB. The biology and pathology of selected skull base tumors. J Neurooncol 1994;20(3):213-240

11 Kadish S, Goodman M, Wang CC. Olfactory neuroblastoma. A clinical analysis of 17 cases. Cancer 1976;37(3):1571-1576

12 Couto HG, et al. Neuroblastoma olfatório ectópico: estudo de caso e revisão da literatura. Rev Med Minas Gerais 2013;23(4): 540-543

13 Araújo. Roberta de Paula et al. Tumores nasossinusais raros: série de casos e revisão de literatura: Relato de caso. Revista Brasileira de Otorrinolaringologia, v. 74, n. 2, p.307-314, 2008.

14 Gil-Carcedo E, Gil-Carcedo LM, Vallejo LA, de Campos JM. Tratamiento del estesioneuroblastoma. Revisión de la literatura. Acta Otorrinolaringol Esp 2005;56(9):389-395

15 Muñoz F, Tresserras P, Montserrat JR, Sancho FJ, Bartumeus F. Estudio retrospectivo de 11 casos de estesioneuroblastomas tratados en el Hospital Santa Creu i Sant Pau entre los años 2000 y 2008 más revisión de la literatura. Neurocirugia (Astur) 2011;22(5):401-417, discussion 417-418

16 Shukla RC, Singh PK, Senthil S, Pathak R. Esthesioneuroblastoma: a case report. Nepal Med Coll J 2010;12(2):128-132

17 Pedruzzi PAG, et al. Estesioneuroblastoma - análise dos casos tratados no Hospital Erasto Gaertner no período de 1973 a 2004. Rev Bras Cir Cabeca Pescoco 2009;38(4):261-263

18 Patel SG, Singh B, Stambuk HE, et al. Craniofacial surgery for esthesioneuroblastoma: report of an international collaborative study. J Neurol Surg B Skull Base 2012;73(3):208-220 